Document downloaded from:

http://hdl.handle.net/10251/65060

This paper must be cited as:

Alcázar-Ortega, M.; Calpe Esteve, CM.; Theisen, T.; Carbonell-Carretero, J. (2015).

Methodology for the identification, evaluation and prioritization of market handicaps which prevent the implementation of Demand Response: Application to European electricity markets. Energy Policy. 86:529-543. doi:10.1016/j.enpol.2015.08.006.

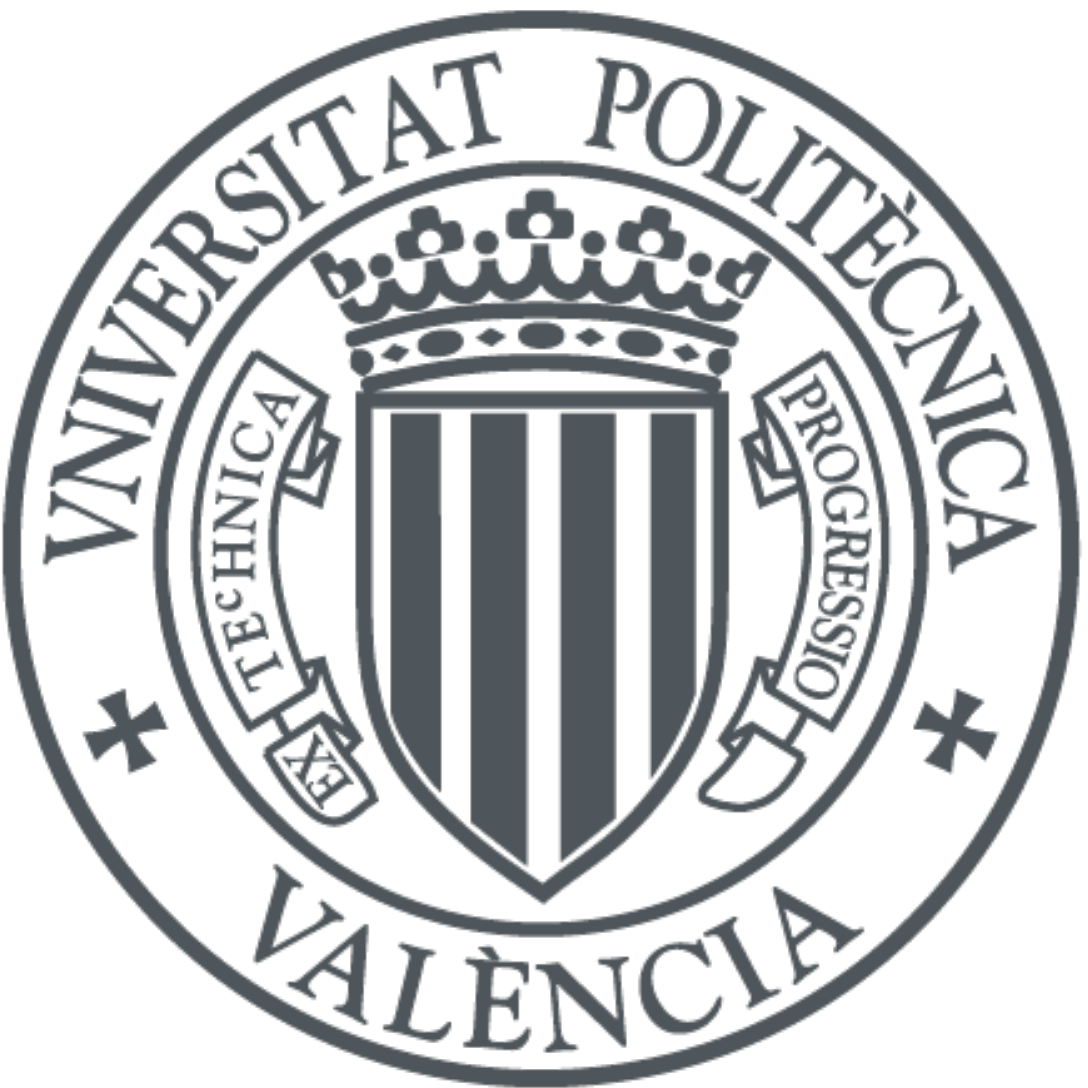

The final publication is available at

http://dx.doi.org/10.1016/j.enpol.2015.08.006

Copyright Elsevier

Additional Information 


\title{
Methodology for the identification, evaluation and prioritization of market handicaps which prevent the implementation of Demand Response: Application to European electricity markets
}

\author{
Manuel Alcázar-Ortega ${ }^{\mathrm{a}, 1,2}$, Carmen Calpe ${ }^{a}$, Thomas Theisen ${ }^{\mathrm{a}}$, José Francisco Carbonell- \\ Carretero $^{\mathrm{b}}$

\begin{abstract}
${ }^{a}$ RWE Deutschland AG, Department of New Technologies, Kruppstraße 5, 45128 Essen, Germany
${ }^{b}$ Universidad Politécnica de Valencia, Institute for Energy Engineering, Camino de Vera, s/n, edificio 8E, escalera $F, 5^{a}$ planta, 46022 Valencia, Spain
\end{abstract}

\section{Keywords}

Demand response; market handicaps; renewable integration; industrial production; energy regulation; load management

\begin{abstract}
This paper presents a methodology for the identification, analysis and comparative assessment of the handicaps which nowadays prevent the higher implementation of Demand Response (DR) in the electricity market. Its application provides a hierarchical organization of handicaps from the most critical to the less critical and then, from the easiest to the most difficult to overcome. This makes possible to determine which barriers would be a priority, which may indicate the direction of regulatory changes to properly address these handicaps and so, stimulating a higher participation of the demand side in electricity markets. After applying the methodology to three European countries, 34 handicaps have been identified, analysing which of these handicaps affect such stakeholders as grid operators, retailers and customers and how these stakeholders are affected. For each handicap, the criticality and difficulty to overcome the different handicaps have been studied, based on detailed information

\footnotetext{
${ }^{1}$ Corresponding author. E-mail addresses: manuel.alcazar-ortega@rwe.com (M. Alcázar-Ortega), carmen.calpe@rwe.com (C. Calpe), Thomas.Theisen@rwe.com (T. Theisen), icarbonell@iie.upv.es (J. F. Carbonell-Carretero)

2 Present address: Universidad Politécnica de Valencia, Institute for Energy Engineering, Camino de Vera s/n, edificio 8E, escalera F, 5a planta, 46020 Valencia, Spain. E-mail address: malcazar@iie.upv.es
} 
coming from personal interviews to experts representing the different stakeholders in the electricity trading chain. Regulatory barriers have been identified as the most critical and difficult to overcome. Together with regulatory changes, the promotion of aggregators and the training of customers on DR applications are some of the most significant initiatives.

\section{Introduction}

The use of Demand Response (DR), understood as the ability of energy consumers to modify the power demand from their expected consumption (Alcázar-Ortega, 2011), may benefit many different market agents in the power system, who can use DR mechanisms for different reasons. Among others, Transmission or Distribution System Operators (TSO/DSOs) may use DR resources to increment capacity reserve in their operation area, or retailers could make use of it in order to balance their energy portfolio. DR can also help to integrate renewable energy resources (RES) in electricity systems, whose production depends on the availability of the primary energy resource and it is not usually correlated to the local demand where such generators are placed (Montuori et al, 2014). Most of RES, like wind power generation, depend on variable and unpredictable external conditions and their supply is not directly controllable (Klobasa, 2009). Therefore, the expansion of renewable energy sources requires new means of load management and greater quantities of various ancillary services (Cappers et al, 2013). In this context, DR aims at adjusting the electricity demand to the grid requirements at a given point of time, meaning an adequate option for reserve capacity.

Several examples worldwide show the interest in increasing the share of RES in the national electricity mixes. Thus, state governments and federal initiatives such as the production tax credit and others have promoted the growth of renewables in the United States (Cardell and Anderson, 2015). In Australia, some research has demonstrated that wind power producers can employ DR to maximize its profit (Mahmoudi et al, 2015). At the other side of the World, in Europe, the promotion of electricity from renewables is a key European Union priority for several reasons, including the security and diversification of energy supply, environmental protection and social and economic cohesion. It makes DR an important pillar for achieving the EU's 20/20/20 goals, which pursue by 2020 not only to raise the share of energy consumption produced from RES to 20\% (European Commission, 2008), but also: 
- Reducing the greenhouse gas emissions in $20 \%$ from 1990 levels, which can be achieved if demand packages are shifted to periods during which less contaminant technologies are producing power (Alcázar-Ortega, 2012).

- Increasing a $20 \%$ the energy efficiency in the Union. As discussed in (Strbac, 2008), DR can contribute to increase the efficiency of the power system due to the relatively low utilisation of generation and networks.

Different provisions dealing with demand side participation have been included in various EU policy documents, specifically the Electricity Directive (2009/72/EC) and the Energy Efficiency Directive (2012/27/EU). On the other hand, a large potential of DR resources have been identified in Europe (Gils, 2014). However, as stated by (Torriti et al, 2010), the market designs and policies in place in most of the European countries do not promote today the participation of demand in electricity markets. On the contrary, DR and related activities such as aggregation remain illegal in many of them and in the majority of system services and wholesale markets (SEDC, 2013).

As it was mentioned above, DR has proven to be a useful mechanism that produces significant benefits for both the customer and the power system (Alcázar-Ortega, 2011), (Shariatzadeh et al, 2015). Customers can obtain significant benefits from DR with the proper price framework (Dupont et al, 2014), (Ghazvini et al, 2015) and DR may bring new business model opportunities for the different stakeholders involved in the trading of this kind of resources (Behrangrand, 2015). However, in spite of the benefits that DR may bring to the society (Alcázar-Ortega, 2011), (US Department of Energy, 2006), allowing a more efficient use of system assets and resources (O'Connell et al, 2014), a number of market handicaps keeps preventing nowadays the massive implementation of DR resources in electricity markets.

The evaluation of market handicaps is not an easy task due to the many different factors to be accounted. Different research examples about market barriers which prevent nowadays the further implementation of DR in the market can be found in the scientific literature. Thus, market barriers for DR implementation in the United States for the provision of ancillary services are studied in (Cappers et al, 2013) and (Greening, 2010). Similarly, other structural and regulatory barriers for the NorthAmerican power system are considered in (Kim and Shcherbakova, 2011). In Europe, barriers for the participation of aggregators in the German balancing market are considered in (Koliou et al, 2014). Moreover, similar examples applied to other electricity markets like Denmark (Katz, 2014), China 
(Zhang et al, 2015) and (Wang et al, 2010), or Austria (Prüggler, 2013) can be found. However, even if there are some examples of quantitative analysis for energy efficiency purposes (Trianni et al, 2013), (Luthra, 2015), no previous research is found regarding market handicaps applied to DR which clearly prioritize which should be the first barriers to be addressed, or how critical may be for the electricity market to do it according to one direction or another. Therefore, a methodology for the identification, evaluation and prioritization of market handicaps which prevent the implementation of Demand Response has been developed and it is here presented. Indeed, the added value of this methodology resides on the systematic evaluation of these barriers, obtaining a hierarchical organization of handicaps according to their criticality and difficulty to solve them. In addition, the responsible to solve each handicap is identified and some recommendations to overcome the different barriers are also given.

This methodology has been designed and applied in the framework of the European LIFE+ Project "Demand Response in Industrial Production" DRIP (Demand Response in Industrial Production "DRIP", 2015). The DRIP consortium was composed of partners from Germany, Spain and the Netherlands, including end-users, distribution system operators (DSOs) and retailers in a way that the whole energy chain was represented. Due to this fact, first-hand information has been used to apply the aforementioned methodology, producing a realistic picture about the market barriers which can be currently found in different places of Europe, including regions from the south-west to the north-east of the continent.

The paper is organized as follows: Section 2 describes the methodology developed for the evaluation of market handicaps on DR. The results of the application to the above mentioned European markets for consumers, system operators and retailers are detailed in Section 3. After that, the results of this research are discussed in Section 4 and finally, the most significant conclusions are summarized in Section 5.

\section{Methodology for the evaluation of market handicaps for DR implementation}

The methodology here presented is schematically shown in Figure 1. This methodology is based on a sequence of steps, as it is detailed in the following paragraphs, which include the gathering of information, the organization of this information in a matrix (MHM), the quantification of the different concepts which define each handicap (such as the category, criticality or difficulty to overcome) and 
the final aggregation and sorting, resulting in a hierarchy of actions to prioritize the solutions of the identified barriers.

\subsection{Definition of the list of handicaps and organization in the market handicaps matrix}

The first step of the developed methodology is the identification of the list of handicaps which may affect the different stakeholders. This identification should be done by means of market analysis and direct contact with different stakeholders in the area where the methodology would be applied. In the case of the DRIP project, the handicaps list was defined based on specific interviews to 57 stakeholders representing the different market roles under study (industrial and commercial customers, DSOs, power producers, energy services companies (ESCOs), retailers, experts on energy regulation, certifiers and aggregators).

Then, the market handicaps need to be placed in a matrix where each one of them is linked to the affected stakeholders. This market handicaps matrix (MHM), whose general structure is presented in Figure 2, contains the following information for each handicap:

- The criticality of the handicap for the considered stakeholder. This factor, as detailed in section 2.3, is graded in a scale of 5 levels: highly critical, medium high, medium low, little critical or almost uncritical.

- The easiness or difficulty to overcome each handicap, also in a grading scale of 5 levels (see section 2.3): very difficult, difficult, moderate, easy or very easy to be overcome.

- The category under which the stakeholders are affected by each handicap. As it is explained more in detail in section 2.2, six categories have been considered: Technical, economic, environmental, psychological/know how, regulatory and organizational. More than one category could apply for each handicap and with different criticality and difficulty to overcome.

- The way in which each handicap can be overcome. An explanation, including some recommendations, should be given for each handicap and stakeholder on how to solve such barrier. These recommendations are essential for the further analysis of potential solutions to minimize the impact of the handicaps under evaluation.

- Finally, the agent who would be responsible to solve each handicap is also identified. This responsible could be the same stakeholder affected by the handicap or a different one (e.g. when technical barriers affecting the customer can be solved by aggregators). 


\subsection{Categories}

Each handicap can affect a market stakeholder from a different perspective (technical, economic, regulatory, etc.). This aspect has been considered by defining a set of categories under which the criticality and difficulty to overcome for each handicap have been organized. The categories defined for this purpose are the following:

1. Psychological/ Know-How. This category includes issues related to the knowledge or background of the technical staff and employees of the stakeholder, abilities to implement or deal with DR issues, training, etc.

2. Technical. This category comprises aspects related to limitations of existing technology, development or application of new technical solutions, equipment for control and management, etc.

3. Economic. Such variables as the costs related to flexibility (investments, labour, cost of monitoring, etc.) and benefits connected to the application of DR (incomes from the participation in some kind of operation service, etc.) are included here.

4. Environmental. Some handicaps could be linked to such issues as emissions into the atmosphere; waste produced in some industrial processes, etc. which could prevent the implementation of some kind of DR action. If so, they are grouped under this category.

5. Regulatory. This kind of barriers is related to the current legal framework regarding the implementation of DR (lack of regulation, opposition of current regulation to the application of DR actions, need of new roles in the market, etc.)

6. Organizational. Finally, this category considers the need of adaptation, change or development of the required infrastructure of companies so as to be ready for DR implementation.

The information for the evaluation of the market handicaps needs to be gathered in interviews to different stakeholders whose activity takes place in the area under study. Later, the information has to be processed and aggregated in order to obtain a single value to quantify the criticality and difficulty to overcome of each handicap. Therefore, a weight could be assigned to each category for aggregation purposes, as it is detailed in section 2.5 .

During the DRIP project, a different importance and, therefore, a different weighting factor was considered for the different categories discussed in this section. These weighting factors, evaluated 
with information gathered in a survey to the different stakeholders are summarized in Table 1.

However, in general, the factors considered for the categories could be included in the weighing of the criticality and difficulty to overcome (Table 2). In such case, factors in Table 1 would be considered equal to 1 and the methodology could be applied in the same way.

\subsection{Criticality and difficulty to overcome}

The criticality and difficulty to overcome are two key parameters which are intrinsically linked to the meaning of market handicaps: a handicap is critical insofar as it hinders or precludes the implementation or the acceptance of DR into the market.

The criticality of each handicap has been categorized under one of the following labels: Highly critical, medium high, medium low, little critical and almost uncritical.

Similarly, the difficulty to overcome quantifies the necessary effort to handle each barrier in order to minimize or counteract its effect. According to the proposed methodology, these levels are defined by the following labels: Very difficult, difficult, moderate, easy and very easy to overcome.

Each criticality and difficulty to overcome factor is linked to a weigh in order to allow the quantification and aggregation of the different parameters related to each handicap, as it is explained in section 2.5. Thus, Table 2 shows the weights considered for each factor according to the values assessed in DRIP.

As previously stated, handicaps may have different values of criticality and difficulty to overcome under several categories. It means that, for example, one handicap could be very critical from a technical point of view while the same handicap may be little critical under an economic perspective.

\subsection{Responsible stakeholder and recommendations}

The assignation of factors is completed with the recommendations or guidelines which should follow each stakeholder in order to overcome the related handicaps. Together with these recommendations, it is also necessary to identify the agent who would be responsible to solve each handicap. In the particular application of this methodology to the mentioned stakeholders in Germany, Spain and the Netherlands, as detailed in section 3, the responsible agent who would be in charge of solving each market handicap has been one of the following:

- The affected stakeholder itself

- The grid operator, when it is different from the affected stakeholder. 
- The retailer or energy trader, when it is different from the affected stakeholder

- The customer or end-user, when it is different from the affected stakeholder

- The aggregator

- The regulator

- The customer with the support of a specialized energy services company (ESCO)

- The aggregator, together with the regulator.

It is important to point out that each handicap has been evaluated according to the answers of different stakeholders, so that this evaluation has been done not just by considering the answers from the stakeholder who is affected by the handicap but also from other stakeholders, including who has to provide the solution.

\subsection{Aggregation and handicaps evaluation}

Each handicap is evaluated by considering the information collected in a number of interviews per stakeholder, as well as different interviews to different stakeholders in order to get an overview about each handicap as complete as possible. On the other hand, one stakeholder can be affected by one handicap under more than one category, as explained in section 2.2. Therefore, it is necessary to aggregate this information in order to get a single value per handicap and stakeholder. According to the answers to these interviews and the assignation of factors to the handicaps under the different categories and stakeholders, it is necessary to perform a numerical evaluation for the criticality and difficulty to overcome of each handicap considering the contribution of the different variables. This contribution is assessed based on the weights assigned to each factor and category. Therefore, the evaluation of each handicap is calculated according to the following expression:

$$
I H_{i-x}=\frac{\sum_{j=1}^{n} W h_{j} \cdot \frac{\sum_{k=1}^{p} W h_{i j k}^{\text {matrix }} \cdot W s_{k}}{\sum_{k=1}^{p} W h_{i j k}^{b i t} \cdot W s_{k}}}{\sum_{j=1}^{n} W h_{i j}^{b i t}}
$$

Where:

- $W h_{j}$ is the weight, from 0 to 1 , assigned to the category $j$ in Table 1.

- $W s_{k}$ is the weight of the interview $\mathrm{k}$, equal to $1 / \mathrm{p}$. 
- $\quad W h_{i j k}{ }^{\text {matrix }}$ is the weight assigned in the MHM to the handicap $i$, the category $j$ and the interview $k$ regarding its criticality and difficulty to overcome, according to the label indicated for each handicap in Table 2.

- $\quad W h_{i j k}^{\text {bit }}$ is a binary variable for each handicap $i$, category $j$ and interview $k$ that is equal to 1 if there is an entry different from zero in the matrix and 0 otherwise.

- $W h_{i j}^{\text {bit }}$ is a binary variable for each handicap $i$ and category $j$ that is equal to 1 when $W h_{j}>0$ for the handicap $i$, and 0 otherwise.

- $\quad n$ is the number of categories (in this case $\mathrm{n}$ is equal to 6 )

- $\quad p$ is the number of interviews per stakeholder

Expression (1) is used to calculate both the global criticality and difficulty to overcome for each handicap, depending on the value taken from Table 2. So, the subscript $x$ indicates whether the index $I H_{i-x}$ is referred to the calculation of the criticality (then $x$ is equal to " $c$ ") or to the difficulty to be overcome ( $x$ takes the value " $d$ ").

\section{Results}

The methodology presented before has been applied to three stakeholders involved in the trading of DR resources in Germany, Spain and the Netherlands. This application is aimed at assessing how these stakeholders could be affected by the identified handicaps and, what is more important, how these handicaps could be overcome (by the stakeholder itself -alone or with the support of some auxiliary entity- or by any other agent). In this particular application, the considered stakeholders have been grid operators (TSO/DSO), retailers and industrial customers. The necessary information was obtained in specific interviews including not only the affected stakeholders, but also other related agents (e.g. who would be responsible to solve the identified handicaps), such as experts in regulation and energy markets.

As a result, a total of 34 handicaps were identified.15 handicaps were related to the system operator, 3 to the retailer and 28 to the customer. The sum of handicaps per stakeholder is not equal to the total number of handicaps since, as explained above, some of them affect to more than one stakeholder.

\subsection{Market handicaps which affect the customer}

The chart in Figure 3 represents the location of the identified market handicaps for industrial and commercial customers, where the variable represented in the abscises axe denotes the difficulty to 
overcome ( 0 for very easy and 1 for very difficult) while the corresponding ordinate is the criticality ( 0 for almost uncritical and 1 for highly critical).

According to Figure 3, the most critical handicaps for customers are usually, at the same time, the most difficult to overcome. In addition, most of them (denoted in the chart with a filled circle) are related to the current regulation, being necessary that regulators (or the regulator together with aggregators) deal with them. In total, almost the $25 \%$ of handicaps need to be solved by the tandem regulator-aggregator.

On the other hand, overcoming the easiest to handle handicaps mostly depends on the customer itself (represented by filled diamonds). They represent about other $25 \%$ of the identified handicaps. Finally, in the middle, there are other handicaps with an intermediate criticality which should be solved by the customer, usually supported by a specialized energy services company (ESCO).

Figure 4(a) shows the number of handicaps classified per category. As discussed previously, some handicaps could be related to more than one category and, in that case, they may be accounted several times. In the case of customers, a half of the total number of handicaps (45\%) is related to technical and know-how reasons (half and half). On the other hand, environmental reasons affect just a little the implementation of DR actions on the customer side. Regulatory barriers represent the $15 \%$ of the total but, as it has been stated before, they are the most critical and most difficult to overcome. This aspect can be seen in Figure 4(b), where the most critical handicaps are accounted. On the other hand, Figure 4(c) shows the most difficult handicaps to overcome. In both cases, regulatory barriers are the most frequent.

Together with regulatory barriers, know-how and economic handicaps have been identified as the most critical for customers. However, the difficulty to overcome such barriers is lower as they could be easily handled with training and the proper analysis of the cost-benefit when applying DR strategies. The handicaps hierarchy is shown in Table 3. Here, handicaps are organized from the most to the less critical and, in the same criticality level, from the easiest to the most difficult to overcome, according to the weighting assigned in the MHM. Consequently, handicaps with the highest criticality and less difficulty to overcome should be the first to be addressed, especially if the solution depends on the customer itself. 


\subsection{Market handicaps which affect the retailer}

Three handicaps affecting the retailer for the implementation of DR have been identified (Figure 5). Two handicaps are medium highly critical while moderately difficult to overcome. A third handicap has been graded as critical but it could be solved by the retailer itself with a low difficulty. Even if not too many handicaps have been identified, it seems clear that the aggregator may play an essential role in the solution of barriers which affect the retailer. Actually, aggregators would be responsible to build significant demand offers by putting together smaller demand packages which may be presented by small and medium industrial and commercial customers.

Considering the categories, the handicaps which affect the retailer are mainly technical and organizational. Regarding the criticality and difficulty to overcome for each category, the most critical barrier is technical. Other handicaps related to technical and organizational reasons are medium highly critical. Regarding the difficulty to overcome, a technical barrier has been identified as the most difficult to overcome, although the difficulty is moderate. On the opposite side, organizational barriers have been denoted as easy or very easy to overcome.

Table 4 shows the handicaps hierarchy for the retailer side, built according to the same criteria as explained in section 3.1 .

\subsection{Market handicaps which affect the system operator}

15 handicaps which affect the system operator have been identified. According to the chart represented in Figure 6, most of these handicaps (60\% of total) should be solved by the regulator. However, the criticality and difficulty to overcome the different handicaps is not clearly linked to who has to solve them. The most critical handicaps (and most difficult to overcome) have to be addressed by the regulator. However, other regulatory barriers are easier to overcome while their criticality is also low. Aggregators and the own grid operator are other agents giving solution to some of the handicaps.

Figure 7 (a) shows the number of handicaps classified per category. For system operators, $60 \%$ of handicaps are economic and regulatory (half and half). On the other side, similarly to the other stakeholders, environmental reasons affect just a little the implementation of DR actions (just $3 \%$ of barriers are classified under this category). Organizational, technical and know-how barriers are in the middle of the table (between $10 \%$ and $15 \%$ ). 
Regarding the criticality, the most critical handicaps are regulatory, although they just represent the $8 \%$ of the identified barriers. Together with regulatory barriers, economic handicaps ( $25 \%$ in total) are the next ones of this scale with an intermediate criticality. As it is shown in Figure $7(\mathrm{~b}), 35 \%$ of handicaps are medium high critical while the criticality of most of the remaining handicaps is low. Most of the barriers are medium critical (high and low), amounting a total of $73 \%$. Like in other studied stakeholders, the less critical barriers are related to environmental issues. Considering the difficulty to overcome, regulatory barriers are the most difficult to handle but no handicaps have been identified as very difficult to overcome. As it is shown in Figure 7(c), most of barriers have a moderate difficulty. The $30 \%$ of barriers are regulatory, being difficult or moderate to overcome. Economic barriers are the second more difficult ones to overcome with moderate difficulty. Table 5 shows the market handicaps hierarchy for the system operator.

\section{Discussion}

As it has been presented, the analysed market handicaps may affect customers, retailers and system operators, but the solution could come from the affected entity or also from a different stakeholder. This section discusses the implications that market handicaps could represent for all of them, including some considerations about how the stakeholders may apply the recommendations mentioned above in order to minimize or counteract the studied barriers. Thus, recommendations are given not just to the affected stakeholders by the market handicaps, but also to the market agents which would be responsible to solve these barriers. Policy implications are related not only to regulatory changes (which are necessary as stated below), but also to internal policies regarding energy efficiency, organizational issues or energy management, which should be internally developed, adopted and applied by the different stakeholders within their own business.

These results have been obtained by applying the methodology to stakeholders from Germany, Spain and the Netherlands, so that the application of these measures would be limited to these three countries.

\subsection{Customers}

DR can become for customers a useful mechanism to increment the competitiveness in the industry as an additional income, once efficiency principles have been applied to processes (Alcázar-Ortega et 
al, 2012). However, there are two issues which need to be properly addressed in order to get industrial customers involved:

- First of all, customers need evidences about the economic profitability of DR in their facilities.

- Secondly, customers need proofs to demonstrate that their processes will not be negatively affected when DR strategies are applied.

These two aspects need to be addressed by means of a detailed energy study (as suggested in such handicaps as A7, A9 or A13 -see Table 3) where the flexibility of processes of a particular customer is identified and the application of DR strategies is evaluated not only technically, but also economically and environmentally. This initial study, which may be performed by an ESCO, is a key in order to solve many of the identified market handicaps, specially related to economic and know-how issues. Complementary training on how to use this flexibility (handicaps A15, A18, A19 or A21) is the other pillar to solve many of the current handicaps from the customer side. This training is essential in order to enable the technical staff of industrial sites to properly apply, evaluate and analyse the impact of DR actions in their facilities. Training may be provided by a specialized ESCO directly working with the head of maintenance of the factory, who would be the connection link to the rest of employees. On the other hand, monitoring of critical parameters driving production processes is essential in order to keep them under control (handicaps A12, A15, or A17). It requires the application of appropriate automatic control so as to get reliable flexibility actions and reduce the necessary notification time in advance. The proper planning to locate control devices and power meters where they are more useful for DR purposes may minimize the required investments (handicap A26).

Finally, an internal policy for DR implementation should be established (handicaps A13, A14, A22 or A25), evaluating the results on DR periodically and the impact of changes in facilities on their flexibility potential. As part of this policy, an internal organization for DR should be defined, assigning tasks and obligations for each workstation from the DR point of view, complementing the existing structure for energy efficiency (if it is the case) or creating a similar one for DR purposes.

\subsection{Retailers}

For retailers, DR would mean an additional option to reduce their costs on the imbalance market. In the case of the use of small demand packages for DR purposes, aggregators (pooling the flexibility of end-users) should develop and execute this service, being reimbursed for delivering the service. Hence, for most of handicaps that impede the development of DR on the retailer side, aggregators 
perform a starring role (handicaps B2 and B3 -see Table 4), building a new business by valorising the flexibility of small industrial customers. In this setting, the retailer is a customer for the aggregator, buying the service of flexibility.

Retailers have a special interest in removing these barriers as they impede them to reduce imbalance costs. When small customers offer their flexibility to the retailer, most of barriers that hinder DR (especially technical and organizational) should be addressed by the aggregator as the natural party to handle these issues (handicaps B2 and B3). In case of larger amounts of flexible power involving just one single customer, handicaps could be directly handled by the retailer itself, directly negotiating with the customer (handicap B3).

On the other hand, barriers which affect the participation of customers on DR issues (Table 3) indirectly affect also to the retailer. When customers overcome their doubts (handicaps A15 or A18), then it is not only the company itself (and the aggregator in case of small customers) who benefit, but also the retailer could benefit since these parties are potential users of the flexibility. This also holds for market handicaps related to the actual service being offered, such as contracting and accurately evaluating the interruptibility capacity. The retailer can also voluntarily offer its expertise to the aggregator in finding a proper solution.

Finally, the contribution of regulators for facilitating the development of the required contractual framework for the trading of demand resources (handicap B3) between customers and retailers (with the intermediation of aggregators for smaller loads) is also requested.

\subsection{System operators}

The most critical barrier for the integration of DR in the power system from the system operator point of view is the lack of aggregators to incorporate small and medium size flexible loads into the system (handicap C1 -see Table 5). Thus, the role of aggregator needs to be promoted by means of the proper regulatory changes, which would enable the participation of such DR resources in operation services. Together with this issue, regulation should be also adapted in order to allow the participation of customers in reserve energy markets in the same conditions as generators.

Due to the number of players in the market, the improvement of communication procedures is also essential to facilitate a higher penetration of DR into the system (handicap C9). The development of standard contracts determining who is allowed to do what and in which conditions is a key in this new scenario (handicaps C4 and C5). 
DR in industrial consumers can help to optimize the management of transmission and distribution networks. In many countries, demand growth is still the main driver for grid expansion needs, where DR is an intelligent option to reduce the related costs. Additionally, new elements such as distributed generation and storage devices or electric vehicles need to be integrated in the distribution grid and operators should assume this new role. Therefore, the definition of operation strategies considering the demand side as an adequate tool to contribute in the grid operation should be enhanced. Finally, grid operators should develop models to consider the behaviour of customers' recovery after the application of flexibility actions so as to evaluate the impact when using such operation tool (handicaps C10 and C11). The specification of these models in existing or new Grid Codes should be considered.

\subsection{Regulators}

It is widely accepted that DR is a form of balancing capacity and, as concluded by (EG3, 2015), it can allay the concerns of some governments and regulators that the future mix of electricity generation capacity delivered by the market may not meet demand at optimal costs. However, as stated in (SEDC, 2013), electricity regulation has been historically written assuming that just generation resources, and not flexible DR resources, could provide the power system with such balancing capacity (handicaps A10, C2 or C15). This fact has blocked the ability of customers to participate because requirements have been designed according to power plant limitations. Consequently, changes should start by allowing energy consumers to participate in reserve energy markets in the same conditions as generators as they have demonstrated to be able to provide the system with reliable balancing services. Moreover, the certification of customers technically able to provide the system with such operation services may be highly recommended and a standard on DR as suggested in DRIP (Demand Response in Industrial Production "DRIP", 2015) should be developed. The lack of standards and regulation (handicap A17 or C7) is also a highly critical barrier. DR cannot be certified today. Discussed the convenience of certification for the different activities involved in the trading of DR resources, an extension of the standard ISO 50.001 could be a useful path to allow the certification of DR in addition to Energy Efficiency.

New regional flexibility markets or alternative non-discriminating measures for the management of distribution networks have to be developed, increasing the economic opportunities of industrial consumers to participate in this kind of services (handicap C2). 
Finally, due to the need of an intermediate entity to use DR for medium size commercial and industrial customers and to offer these capacities to the market, appropriate regulation is needed to enable the role of aggregators (handicaps $\mathrm{A} 3$ or $\mathrm{C} 1$ ). This still has to be developed.

\subsection{Aggregators}

The role of aggregators in the electricity market should be promoted to get small and medium industrial and commercial customers dynamically involved (handicaps A3 and C1). Communication channels between metering devices at consumer facilities and aggregators need to be improved, reducing the time between measurements and facilitating the validation and assessment of DR actions depending on the type of load (handicaps A20, C1 or C20). The use of Advanced Metering Infrastructure (AMI) should be highlighted as a solution, especially when European countries are required to ensure the implementation of smart metering under EU energy market legislation (European Commission, 2014).

Specific methods to validate the performance of DR actions (baseline) need to be defined, considering the particularities of each DR program and the characteristics of the specific processes (handicaps $\mathrm{A} 12$ or B2).

Finally, it should be highlighted the need of new tools oriented to analyse and optimize the benefit of DR providers (handicap B1) in order to stimulate the participation and involvement of consumers.

\section{Conclusions and Policy Implications}

A methodology for the evaluation of the handicaps which may prevent the implementation of DR techniques in the market has been developed and it is presented here. This methodology has been applied under the scope of the European DRIP project to the electricity markets in Germany, Spain and the Netherlands. As result, different barriers have been analysed, prioritized and classified according to their criticality and difficulty to overcome for the different stakeholders affected by each handicap, providing recommendations about potential solutions and the guidelines to whom would be responsible to address each of them. Keeping in mind that these results are limited to the scope of the DRIP project in the three considered countries, as well as that the input information is coming from expert opinions, a detailed analysis of market handicaps which affect customers, grid operators and retailers for the implementation of DR has been carried out. The main outputs of this analysis can be highlighted as follows: 
- A total of 34 handicaps (outspread into 46 items since some of them affect more than one stakeholder) which affect to customers, retailers and grid operators can be accounted. A number of 28 handicaps has been identified for customers, 3 for retailers and 15 for system operators.

- The most critical handicaps and, at the same time, the most difficult to be overcome, have to be solved by the regulator. The regulator is also who has to solve most of the identified handicaps with a $34 \%$ of total. The $8 \%$ of handicaps identified in customers are regulatory, while for system operators they mean the $30 \%$ of barriers.

- Considering all the stakeholders together, economic barriers are the most significant in number, followed by regulatory. Handicaps with the highest criticality affect mainly customers, which are actually affected by the 9 most critical handicaps.

In particular, customers consider regulatory barriers as the most critical and the most difficult to overcome, while the easiest barriers to handle are related to economic and know-how issues. A detailed energy study to identify and evaluate the flexibility of the involved facilities is essential since customers would be willing to participate in DR services just if they have evidences about the economic profitability of such services while their processes are not affected when DR strategies are applied.

For the retailer perspective, DR is an additional option to reduce costs on the imbalance market. Most of the barriers which affect to the retailer are technical and organizational, although economic matters are the most difficult to overcome.

From the system operator perspective, most of barriers should be solved by the regulator. Together with regulatory barriers, economic handicaps are also critical for system operators. One of the most critical barriers is the lack of aggregators to incorporate small and medium size flexible loads into the system. The role of aggregator needs to be promoted, which would enable the participation of such DR resources in operation services. Together with this issue, regulation should be adapted in order to allow the participation of customers in reserve energy markets in the same conditions as generators. One of the most interesting results of the proposed methodology is the hierarchical organization of handicaps according to their criticality and difficulty to overcome, based on the weighting assigned to these factors for each handicap. This fact makes possible to determine which barriers would be a 
priority, showing to the different stakeholders the strategy they should follow in order to effectively enable the participation of DR into the market.

Summarizing: Regulatory changes to allow the participation of demand side in balancing services in the same conditions as generators, the enablement and promotion of aggregators for the integration of smaller loads or the training of industrial and commercial customers on Demand Response applications are some of the most important initiatives which, if properly addressed, will establish the bases for a real Demand Response market, contributing to a more efficient power system where customers are fully involved.

\section{Acknowledgements}

This work was completed in the framework of the DRIP project (11ENV/DE/340) co-funded by the European Commission through the LIFE Environment Programme. The authors deeply thank all the participants in the project for their help and support that made possible this work.

\section{References}

Alcázar-Ortega M, 2011. Evaluation and Assessment of New Demand Response Products Based on the Use of Flexibility in Industrial Processes: Application to the Food Industry. PhD Dissertation, Dual PhD Programme between the Polytechnic University of Valencia and the University of South Florida, Tampa, Florida.

Alcázar-Ortega M, Álvarez-Bel C, Domijan A, Escrivá-Escrivá G, 2012. Economic and environmental evaluation of customers' flexibility participating in operation markets: Application to the meat industry. Energy, 41, 368-379.

Behrangrand M, 2015. A review of demand side management business models in the electricity market. Renewable and Sustainable Energy Reviewers, 47, 270-283.

Cappers P, MacDonald J, Goldman C, Ookie M, 2013. An assessment of market and policy barriers for demand response providing ancillary services in U.S. electricity markets. Energy Policy, 62, 1031-1039.

Cardell JB, Anderson CL, 2015. Targeting existing power plants: EPA emission reduction with wind and demand response. Energy Policy, 4, 11-23. 
Demand Response in Industrial Production "DRIP", 2015. Co-funded by the EU LIFE+ Environmental Programme. Retrieved 15/03/2015 from www.drip-project.eu

Dupont B, De Jonghe D, Olmos L, Belmans R, 2014. Demand response with locational dynamic pricing to support the integration of renewables. Energy Policy, 67, 334-354.

EG3, 2015. Regulatory Recommendations for the Deployment of Flexibility. Brussels: European Commission, Smart Grid Task Force. Retrieved 25/02/2015 from https://ec.europa.eu/energy/sites/ener/files/documents/EG3\%20Final\%20\%20January\%202015.pdf

European Commission, 2014. Benchmarking smart metering deployment in the EU-27 with a focus on electricity. Brussels: Report from the European Commission. Retrieved 19/02/2015, from http://eur-lex.europa.eu/legal-content/EN/TXT/?uri=COM:2014:356:FIN

European Commission, 2008. 2020 by 2020 Europe's climate change opportunity. Brussels: Report from the European Commission. Retrieved 12/06/2015, from: http://eur-lex.europa.eu/legalcontent/EN/TXT/?uri=CELEX:52008DC0030

Ghazvini MF, Faria P, Ramos S, Morais H, Vale Z, 2015. Incentive-based demand response programs designed by asset-light retail electricity providers for the day-ahead market. Energy, 82, 786-799.

Gils HC, 2014. Assessment of the theoretical demand response potential in Europe. Energy, 67, 1-8.

Greening L A, 2010. Demand response resources: Who is responsible for implementation in a deregulated market? Energy, 35, 1518-1525.

Katz J, 2014. Linking meters and markets: Roles and incentives to support a flexible demand side. Utilities Policy, 31, 74-84.

Kim JH, Shcherbakova A, 2011. Common failures of demand response. Energy, 36, 873-880)

Klobasa M, 2009. Analysis of Demand Response and wind integration in Germany's electricity market. IET Renewable Power Generation, 4, 55-63. 
Koliou E, Eid C, Chaves-Ávila JP, Hakvoort RA, 2014. Demand response in liberalized electricity markets: Analysis of aggregated load participation in the German balancing mechanism. Energy, 71, 245-254.

Luthra S, Kumar S, Garg D, Haleem A, 2015. Barriers to renewable/sustainable energy technologies adoption: Indian perspective. Renewable and Sustainable Energy Reviewers, 41, 762-776.

Mahmoudi N, Saha TK, Eghbal M, 2015. Wind offering strategy in the Australian National Electricity Market: A two-step plan considering demand response. Electric Power System Research, $119,187-798$.

Montuori L, Alcázar-Ortega M, Álvarez-Bel C, Domijan A, 2014. Integration of renewable energy in microgrids coordinated with demand response resources: Economic evaluation of a biomass gasification plant by Homer Simulator. Applied Energy, 132, 15-22.

O'Connell N, Pinson P, Madsen H, O'Malley M, 2014. Benefits and challenges of electrical demand response: A critical review. Renewable and Sustainable Energy Reviews, 39, 686-699.

Prüggler N, 2013. Economic potential of demand response at household level - Are Central-European market conditions sufficient? Energy Policy, 60, 487-498.

SEDC, 2013. A Demand Response Action Plan for Europe: Regulatory requirements and market models. Brussels: Smart Energy Coalition. Retrieved 17/01/2015 from http://sedccoalition.eu/wp-content/uploads/2013/06/SEDC-DR-FINAL-.pdf

Shariatzadeh F, Mandal P, Srivastava AK, 2015. Demand response for sustainabile energy systems: A review application and implementation strategy. Renewable and Sustainable Energy Reviews, 45, 343-350.

Strbac G, 2008. Demand side management: Benefits and challenges. Energy Policy, 36, 4419-4426.

Torriti J, Hassan MG, Leach M, 2010. Demand response experience in Europe: Policies, programmes and implementation. Energy, 35, 1575-1583.

Trianni A, Cagno E, Worrell E, Pugliese G, 2013. Empirical investigation of energy efficiency barriers in Italian manufacturing SMEs. Energy, 49, 444-458. 
US Department of Energy, 2006. Benefits of demand response in electricity markets and recommendations of achieving them. Report to the United States Congress. Retrieved 05/03/2015 from http://energy.gov/sites/prod/files/oeprod/DocumentsandMedia/DOE Benefits of Demand Re sponse in Electricity Markets and Recommendations for Achieving Them Report to Co ngress.pdf

Wang J, Bloyd CN, Hu Z, Tan Z, 2010. Demand Response in China. Energy, 35, 1592-1597.

Zhang F, Deng H, Margolis R, Su J, 2015. Analysis of distributed-generation photovoltaic deployment, installation time and cost, market barriers and policies in China. Energy Policy, 81, 43-55. 